\title{
Pseudo-Entanglement Evaluated in Noninertial Frames
}

\author{
Hossein Mehri-Dehnavi*, Behrouz Mirza ${ }^{\dagger}$ Hosein Mohammadzadeh; \\ and Robabeh Rahimi ${ }^{\S}$ \\ * Department of Physics, Institute for Advanced Studies in Basic Sciences, \\ Zanjan 45195-1159, Iran, \\ * Research Center for Quantum Computing, Kinki University, 3-4-1 Kowakae, \\ Higashi-Osaka, Osaka 577-8502, Japan \\ $\dagger, \ddagger$ Department of Physics, Isfahan University of Technology, \\ Isfahan 84156-83111, Iran \\ $\S$ Department of Chemistry, Graduate School of Science, Osaka City University, \\ Osaka 558-8585, Japan \\ $\S$ Institute for Quantum Computing and Department of Physics and Astronomy, \\ University of Waterloo, Waterloo, ON, N2L 3G1, Canada.
}

\begin{abstract}
We study quantum discord, in addition to entanglement, of bipartite pseudo-entanglement in noninertial frames. It is shown that the entanglement degrades from its maximum value in a stationary frame to a minimum value in an infinite accelerating frame. There is a critical region found in which, for particular cases, entanglement of states vanishes for certain accelerations. The quantum discord of pseudo-entanglement decreases by increasing the acceleration. Also, for a physically inaccessible region, entanglement and nonclassical correlation are evaluated and shown to match the corresponding values of the physically accessible region for an infinite acceleration.
\end{abstract}

Keywords: quantum computing; entanglement; nonclassical correlation; noninertial frames; ensemble systems

\footnotetext{
${ }^{*}$ E-mail address: mehri@alice.math.kindai.ac.jp

${ }^{\dagger}$ E-mail address: b.mirza@cc.iut.ac.ir

${ }^{\ddagger}$ E-mail address: h.mohammadzadeh@ph.iut.ac.ir

$\S$ E-mail address: rrahimid@uwaterloo.ca
} 


\section{Introduction}

Implementations of quantum information protocols and achievements of any advantage over classical computers have formed the underlying causes for the high popularity of researches on quantum information processing. This is generally true for studies not only in an inertial frame but also for those within noninertial frames when the relative motion and/or the acceleration of the communicating partners are large [1]. Quantum entanglement, mentioned as the essence of quantum physics [2] and believed to be a possible quantum resource for a quantum processor in surpassing the presently available classical computers, has been widely studied theoretically [3] and experimentally, for a variety of physical systems as well as for inertial/noninertial frames [1, 4, 5, 6, 7, 8, 9, 10, 11, 12, 13, 14, 15, 16].

When entanglement is considered in noninertial frames, a relativistic frame should be employed. In principle, an observer with uniform acceleration cannot obtain information about the whole spacetime from his perspective, which leads to a communication horizon to appear. This results in a loss of information and a corresponding degradation of entanglement. It has been shown that the Unruh effect [17] degrades the entanglement between partners [5, 6, 7]. Therefore, the implementation of certain quantum information processing tasks between accelerated partners requires a quantitative understanding of such degradation in noninertial frames. As a natural question, one may ask how the involved parties in a state, particularly in an entangled state, behave in accordance to each other from the viewpoints of the accelerating and resting observers.

The key role of entanglement for quantum information processing, in general, has not been yet proved. The power of a quantum processor has been also mentioned to be due to any existence of correlation other than the purely classical one. Such correlations include, but not restricted, the entanglement of the states. Meanwhile, attempts have been made to revise the distinct role of entanglement since the original work by Ollivier and Zurek [18], stating that a separable state (hence nonentangled by definition) may present quantum correlation [19, 20].

Nonclassically correlated states other than entanglement have shown to present computational powers beyond classical schemes [21. In addition, almost all the implementations of quantum information protocols by conventional nuclear magnetic resonance (NMR) [22, electron nuclear double resonance (ENDOR) [23], or other similar bulk ensemble quantum computers at room temperature have been under experimental conditions of what just could provide a pseudo-pure state as follows

$$
\rho_{\mathrm{ps}}=\frac{1-p}{4} I+p|\psi\rangle\langle\psi|
$$

where, $p$ characterizes the fraction of state $|\psi\rangle$ which has been originally the desired initial simple and fiducial state for quantum computation, $|00\rangle$ [24]. In order to produce a particular entangled state, the corresponding entangling operation $E$ is applied to the pseudo-pure state and changes it to

$$
\rho=\frac{1-p}{4} I+p\left|\Phi^{+}\right\rangle\left\langle\Phi^{+}\right|
$$


where, $\left|\Phi^{+}\right\rangle=E|\psi\rangle=\frac{1}{\sqrt{2}}(|00\rangle+|11\rangle)$. This state is entangled if and only if $p>1 / 3$. Otherwise, for a case of $p>0$, it is separable but yet involves nonclassical correlations. In an experiment involving two spins representing to the two parties $A$ and $B, E$ can be a combination of Hadamard gate on $A$ followed by a CNOT gate on both sides.

Recent trends point toward extending quantum information processing to noninertial frames. Also, once a bulk ensemble system is supposed as a testbed for implementations in either inertial or noninertial frames, the existence and, accordingly, the behavior of the nonclassical correlations are even more important than the status of entanglement. In view of this demand, in this work, we study the pseudo-entangled state (2) for noninertial frames. This can be complementary to previous studies for an inertial frame. However, for the sake of selfcompleteness, results of an overview on the existence of entanglement are given in an appropriate place.

This paper is organized as follows. Section II is to give a short survey of entanglement and nonclassical correlation; for each class a measure is described which is extensively employed in this work. Section III is a short review on the Dirac fields in noninertial frames. Section IV provides the main results of the study. Finally, summary and discussions will conclude the paper.

\section{Classical/Nonclassical Correlations}

Classical/nonclassical correlations for subsystems of a quantum system have been indispensable subjects in quantum information theory. For historical reasons, a nonclassically correlated state may be explained by reviewing the concept of entanglement.

An entangled state, according to separability paradigm [25, 26], cannot be prepared by local operations and classical communications (LOCC) [27]. For a density matrix $\rho$ of a composite bipartite system $A B$, a separable state can be written as follows

$$
\rho_{\mathrm{sep}}=\sum_{i} w_{i} \rho_{A}^{i} \otimes \sigma_{B}^{i}
$$

where, $w_{i}$ 's are positive weights, and $\rho_{A}^{i}$ 's and $\sigma_{B}^{i}$ 's are local states belonging to $A$ and $B$, respectively. An entangled state $\rho_{\text {ent }}$ is an inseparable state that is not of the above form.

There are other paradigms that are mostly based on a post-preparation stage [18, 28, 29, 30] rather than a preparation one. According to Oppenheim-Horodecki paradigm [31], a properly classically correlated state of a bipartite system $A B, \rho_{\mathrm{pcc}}$, is defined as a state represented by

$$
\rho_{\mathrm{pcc}}=\sum_{i=1}^{d^{A}} \sum_{j=1}^{d^{B}} e_{i j}\left|v_{A}^{i}\right\rangle\left\langle v_{A}^{i}|\otimes| v_{B}^{j}\right\rangle\left\langle v_{B}^{j}\right|,
$$

where, $d^{A}$ and $d^{B}$ are the dimensions of the Hilbert spaces of $A$ and $B$, respectively. $e_{i j}$ is the eigenvalue of $\rho_{\text {pcc }}$ corresponding to an eigenvector $\left|v_{A}^{i}\right\rangle \otimes\left|v_{B}^{j}\right\rangle$. Based on this definition, a nonclassically correlated state, $\rho_{\mathrm{ncc}}$, is a state that cannot be described as the above form (4). 
Several approaches are available for evaluating the status of entanglement in a system [32, 33]. Logarithmic Negativity of the state $\rho, N(\rho)$, is a nonconvex entanglement monotone which gives one of the most powerful means for quantifying entanglement. For a bipartite system $\rho, N(\rho)$, is defined as

$$
N(\rho):=\log _{2} \sum_{i}\left|\lambda_{i}\left(\rho^{\mathrm{pt}}\right)\right|,
$$

where, $\lambda_{i}\left(\rho^{\mathrm{pt}}\right)$ denotes the eigenvalues of the partial transpose, $\rho^{\mathrm{pt}}$, of $\rho$. According to the properties of the partial transpose, the logarithmic negativity is symmetric with respect to $A$ and $B$. This behavior is not observed for quantum discord explained below.

As for nonclassical correlations, among the exiting measures [18, 29, 31, 34, 35, 36, 37, 38, 39, 40], we evaluate quantum discord [18], which has been studied for quantum computing [21, 37, 41, 42] and broadcasting of quantum states [43, 44]. The dynamics of quantum discord is also studied [45].

Before we embark on reformulating quantum discord, it is important to note that measures of entanglement and classical/quantum correlations can be conceptually different from each other. For example, since quantum discord is not generally identical with an entanglement measure, any direct comparison of the two notions can be meaningless. For a comparison to be valid and to produce reasonable data, one needs to employ a unified approach [41].

Quantum discord for a bipartite quantum system is the discrepancy between the quantum mutual information and the locally accessible mutual information. These two concepts for measuring the mutual information context are classically identical. The quantum mutual information is defined as

$$
\mathcal{I}(A: B)=S\left(\rho_{A}\right)+S\left(\rho_{B}\right)-S(\rho),
$$

where, $\rho_{A}$ and $\rho_{B}$ are the density operators of $A$ and $B$, respectively. Here, $S(\rho)=-\operatorname{Tr}\left(\rho \log _{2} \rho\right)$ is the von Neumann entropy.

The locally accessible mutual information, $\mathcal{J}$, is represented by

$$
\mathcal{J}_{\left\{\Pi_{k}\right\}}(A: B)=S\left(\rho_{A}\right)-S_{\left\{\Pi_{k}\right\}}(A \mid B) .
$$

Here, $\left\{\Pi_{k}\right\}$ 's are von Neumann operators acting on subsystem $B$ and corresponding to the outcome k. $S_{\left\{\Pi_{k}\right\}}(A \mid B)$ is the quantum conditional entropy, that is

$$
S_{\left\{\Pi_{k}\right\}}(A \mid B)=\sum_{k} p_{k} S\left(\rho_{A \mid k}\right),
$$

where, $\rho_{A \mid k}=\operatorname{Tr}_{B}\left(\Pi_{k} \rho \Pi_{k}\right) / p_{k}$, with $p_{k}=\operatorname{Tr}\left(\Pi_{k} \rho \Pi_{k}\right)$. The classical correlation is given by [18, 19, [20, 36, 46]

$$
\mathcal{C}(A: B)=\max _{\left\{\Pi_{k}\right\}}\left[\mathcal{J}_{\left\{\Pi_{k}\right\}}(A: B)\right] .
$$

By substituting the appropriate values, the quantum discord is calculated as

$$
\mathcal{D}(A: B)=\mathcal{I}(A: B)-\mathcal{C}(A: B) .
$$


One can also select a different set of von Neumann operators, $\left\{\Pi_{k}^{\prime}\right\}$, acting on the subsystem $A$ and corresponding to the outcome $k$, that can be written as $S_{\left\{\Pi_{k}^{\prime}\right\}}(B \mid A)=\sum_{k} p_{k}^{\prime} S\left(\rho_{k \mid B}^{\prime}\right)$; where, $\rho_{k \mid B}^{\prime}=\operatorname{Tr}_{A}\left(\Pi_{k}^{\prime} \rho \Pi_{k}^{\prime}\right) / p_{k}^{\prime}$, with $p_{k}^{\prime}=\operatorname{Tr}\left(\Pi_{k}^{\prime} \rho \Pi_{k}^{\prime}\right)$. Finding the minimum value of $S_{\left\{\Pi_{k}^{\prime}\right\}}(B \mid A)$, over all von Neumann operators, we can evaluate the classical correlation as follows:

$$
\mathcal{C}(B: A)=S\left(\rho_{B}\right)-\min _{\left\{\Pi_{k}^{\prime}\right\}} S_{\left\{\Pi_{k}^{\prime}\right\}}(B \mid A) .
$$

Thus, quantum discord $\mathcal{D}(B: A)$, is obtained as,

$$
\mathcal{D}(B: A)=\mathcal{I}(B: A)-\mathcal{C}(B: A) \text {. }
$$

It has been mentioned by Zurek [47] that quantum discord is not generally symmetric, i.e., we have

$$
\mathcal{D}(A: B) \neq \mathcal{D}(B: A)
$$

In following we will observe this property.

\section{Dirac Fields in Noninertial Frames}

Let us suppose a bipartite system. The resting part is named as Alice, A, and the accelerating part as Rob, R. Alsing et. al. [6] have studied the degradation of entanglement by Unruh effect for the bipartite system. If we consider Rob to be uniformly accelerated in the $(t, z)$ plane, then the appropriate Rindler coordinates $(\tau, \zeta)$ introduce two different Rindler regions that are causally disconnected from each other:

$$
\begin{aligned}
& a t=e^{a \zeta} \sinh (a \tau), \quad a z=e^{a \zeta} \cosh (a \tau), \\
& a t=-e^{a \zeta} \sinh (a \tau), \quad a z=-e^{a \zeta} \cosh (a \tau) .
\end{aligned}
$$

The above set of coordinates is related to regions I and II, respectively, and $a$ denotes Rob's proper acceleration. One can quantize a free spinor field, in $3+1$ dimensions, which satisfies the Dirac equation by using the complete orthonormal set of fermion, $\psi_{k}^{+}$, and antifermion, $\psi_{k}^{-}$, modes,

$$
\psi=\int\left(a_{k} \psi_{k}^{+}+b_{k}^{\dagger} \psi_{k}^{-}\right) d k
$$

where, $a_{k}^{\dagger}\left(b_{k}^{\dagger}\right)$ and $a_{k}\left(b_{k}\right)$ are the creation and annihilation operators for fermions (antifermions) of the momentum $k$, respectively. They satisfy the anticommutation relation

$$
\left\{a_{i}, a_{j}^{\dagger}\right\}=\left\{b_{i}, b_{j}^{\dagger}\right\}=\delta_{i j} .
$$

The quantum field theory for the Rindler observer is constructed by expanding the spinor field in terms of a complete set of fermion and antifermion modes in regions I and II, as already introduced in Eq. (14),

$$
\psi=\int \sum_{\sigma}\left(c_{k}^{\sigma} \psi_{k}^{\sigma+}+d_{k}^{\sigma \dagger} \psi_{k}^{\sigma-}\right) d k, \quad \sigma \in\{\mathrm{I}, \mathrm{II}\}
$$


where, $c_{k}^{\sigma \dagger}\left(d_{k}^{\sigma \dagger}\right)$ and $c_{k}^{\sigma}\left(d_{k}^{\sigma}\right)$ are the creation and annihilation operators for fermion (antifermions), respectively, acting on region I (II) for $\sigma=$ I (II) and satisfying an anticommutation relation similar to Eq. (16). One can find a relation between creation and annihilation operators of Minkowski and Rindler spacetime using Bogoliubov transformation [48, 49, 50.

$$
a_{k}=\cos r c_{k}^{\mathrm{I}}-\sin r d_{-k}^{\mathrm{II} \dagger}, \quad b_{k}=\cos r d_{k}^{\mathrm{I}}+\sin r c_{-k}^{\mathrm{II} \dagger},
$$

where, $\cos r=1 / \sqrt{1+e^{-2 \pi \omega c / a}}$ with $\omega=\sqrt{|\vec{k}|^{2}+m^{2}}$. It is easy to see from Eq. (18) and its adjoint that Bogoliubov transformation mixes a fermion in region I and antifermions in region II. Therefore, we postulate that the Minkowski particle vacuum state for mode $k$ in terms of Rindler Fock states is given by

$$
\left|0_{k}\right\rangle_{M}=\sum_{n=0}^{1} A_{n}\left|n_{k}\right\rangle_{\mathrm{I}}^{+}\left|n_{-k}\right\rangle_{\mathrm{II}}^{-}
$$

As a comment on notation, the Rindler region I or II Fock states carry a subscript I and II, respectively, on the kets, while the Minkowski Fock states are indicated by the subscript $M$ on the kets. In the following, we use the single mode approximation and we will drop all labels $(k,-k)$ on states and density matrices indicating the specific mode. By applying the creation and annihilation operators to Eq. (19) and using the normalization condition, one can obtain [6]

$$
|0\rangle_{M}=\cos r|0\rangle_{\mathrm{I}}|0\rangle_{\mathrm{II}}+\sin r|1\rangle_{\mathrm{I}}|1\rangle_{\mathrm{II}},
$$

and in a same manner

$$
|1\rangle_{M}=|1\rangle_{\mathrm{I}}|0\rangle_{\mathrm{II}}
$$

Also, we can show that when Rob accelerates uniformly through the Minkowski vacuum, his detector registers a number of particles given by

$$
+\left\langle 0\left|c^{\mathrm{I} \dagger} c^{\mathrm{I}}\right| 0\right\rangle^{+}=\frac{1}{e^{2 \pi \omega / a}+1}
$$

where, $\omega$ is related to the specified $k$ mode. Equation (22) is known as the Unruh effect [17], which demonstrates that Rob in region I detects a thermal Fermi-Dirac distribution of particles as he passes through the Minkowski vacuum. It is notable that more recently the Unruh effect beyond the single mode approximation has been considered [51]. 


\section{Discord and Entanglement of Pseudo-Entangled States in Noninertial Frames}

We would like to study the state (2) in noninertial frames. Using Eqs. (20) and (21), we find the relating density matrix as follows

$\rho_{A, \mathrm{I}, \mathrm{II}}=\frac{1}{4}\left(\begin{array}{cccccccc}(1+p) \cos ^{2} r & 0 & 0 & \frac{1+p}{2} \sin 2 r & 0 & 0 & 2 p \cos r & 0 \\ 0 & 0 & 0 & 0 & 0 & 0 & 0 & 0 \\ 0 & 0 & 1-p & 0 & 0 & 0 & 0 & 0 \\ \frac{1+p}{2} \sin 2 r & 0 & 0 & (1+p) \sin ^{2} r & 0 & 0 & 2 p \sin r & 0 \\ 0 & 0 & 0 & 0 & (1-p) \cos ^{2} r & 0 & 0 & \frac{1-p}{2} \sin 2 r \\ 0 & 0 & 0 & 0 & 0 & 0 & 0 & 0 \\ 2 p \cos r & 0 & 0 & 2 p \sin r & 0 & 0 & 1+p & 0 \\ 0 & 0 & 0 & 0 & \frac{1-p}{2} \sin 2 r & 0 & 0 & (1-p) \sin ^{2} r\end{array}\right)$

where, we have used the basis $|000\rangle,|001\rangle,|010\rangle,|011\rangle,|100\rangle,|101\rangle,|110\rangle$, and $|111\rangle$, with $|\operatorname{lmn}\rangle:=$ $|l\rangle_{A}|m\rangle_{\mathrm{I}}|n\rangle_{\mathrm{II}}$, in writing the above matrix.

A bipartite density matrix is obtained by tracing out the modes of one of the regions $A$, I, or II, in which logarithmic negativity and quantum discord are applied for evaluating the status of entanglement and nonclassical correlation, respectively. It turns out that by tracing out each element of $A$, I, or II, the resulting matrix form of the state is a real symmetric $X$-shaped density matrix. Therefore, we review quantum discord for the set of $X$-states in Appendix A.

\subsection{Bipartition Alice-Rob}

For finding the relating density matrix between Alice and Rob, the antiRob's modes of $\rho_{A, \mathrm{I}, \mathrm{II}}$ are traced out, i.e., $\rho_{A, \mathrm{I}}=\operatorname{Tr}_{\mathrm{II}}\left(\rho_{A, \mathrm{I}, \mathrm{II}}\right)$. Using the basis $|0\rangle_{A}|0\rangle_{\mathrm{I}},|0\rangle_{A}|1\rangle_{\mathrm{I}},|1\rangle_{A}|0\rangle_{\mathrm{I}}$, and $|1\rangle_{A}|1\rangle_{\mathrm{I}}$, it can be written as

$$
\rho_{A, \mathrm{I}}=\frac{1}{4}\left(\begin{array}{cccc}
(1+p) \cos ^{2} r & 0 & 0 & 2 p \cos r \\
0 & 1+\sin ^{2} r-p \cos ^{2} r & 0 & 0 \\
0 & 0 & (1-p) \cos ^{2} r & 0 \\
2 p \cos r & 0 & 0 & 1+\sin ^{2} r+p \cos ^{2} r
\end{array}\right) .
$$

The eigenvalues of $\rho_{A, \mathrm{I}}$ are given by

$$
\begin{aligned}
& \lambda_{1,2}\left(\rho_{A, \mathrm{I}}\right)=\frac{1}{4}\left\{1+p \cos ^{2} r \pm \sqrt{4 p^{2} \cos ^{2} r+\sin ^{4} r}\right\}, \\
& \lambda_{3,4}\left(\rho_{A, \mathrm{I}}\right)=\frac{1}{4}\left\{1-p \cos ^{2} r \pm \sin ^{2} r\right\} .
\end{aligned}
$$

Thus, the von Neumann entropy of $\rho_{A, \mathrm{I}}$ is calculated as $S\left(\rho_{A, \mathrm{I}}\right)=-\sum_{j=1}^{4} \lambda_{j} \log _{2} \lambda_{j}$. Similarly, the entropy of the reduced density matrices $\rho_{A}$ and $\rho_{\mathrm{I}}$ are obtained as $S\left(\rho_{A}\right)=1$ and $S\left(\rho_{\mathrm{I}}\right)=$ $-\frac{\cos ^{2} r}{2} \log _{2} \frac{\cos ^{2} r}{2}-\frac{1+\sin ^{2} r}{2} \log _{2} \frac{1+\sin ^{2} r}{2}$, respectively. 


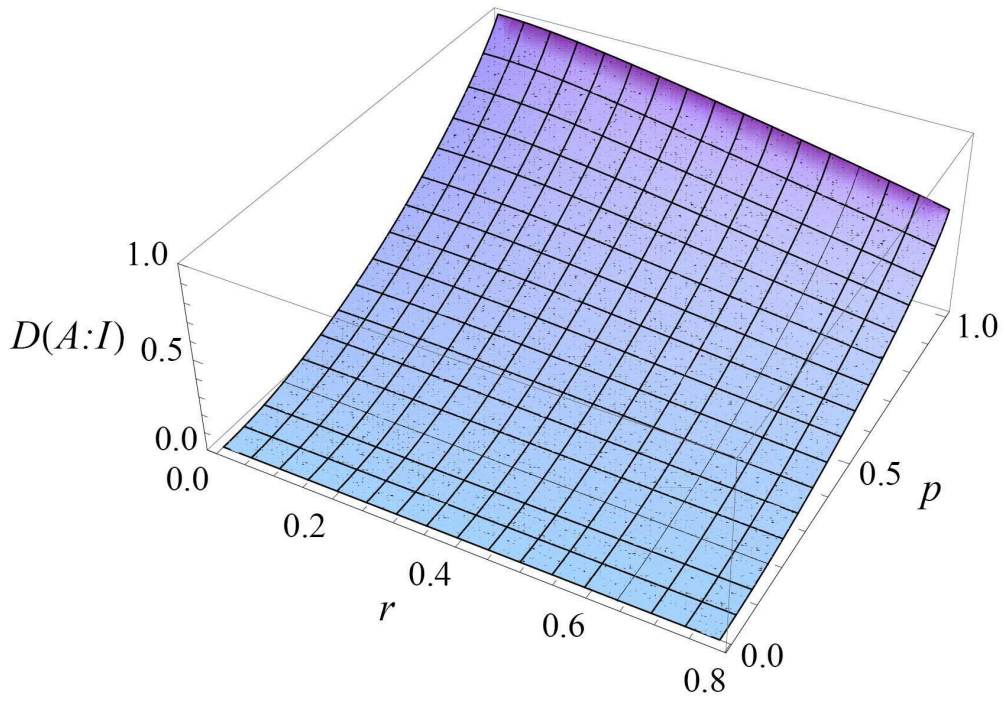

Figure 1: Quantum discord, $\mathcal{D}(A: \mathrm{I})$, as function of $r$ and $p$.

Therefore, quantum discord is given by

$$
\mathcal{D}(A: \mathrm{I})=\min _{\left\{\Pi_{k}\right\}}\left[S_{\left\{\Pi_{k}\right\}}(A \mid \mathrm{I})\right]-S\left(\rho_{A, \mathrm{I}}\right)-\frac{\cos ^{2} r}{2} \log _{2} \frac{\cos ^{2} r}{2}-\frac{1+\sin ^{2} r}{2} \log _{2} \frac{1+\sin ^{2} r}{2} .
$$

It has been discussed in the Appendix that it suffices to simply check four end points. By plotting $S_{\left\{\Pi_{k}\right\}}(A \mid \mathrm{I})$ for these cases, the case $\kappa=\ell=1 / 2$ with $\beta=1 / 4$ is corresponding to this minimum. Replacing this value in Eq. (26) quantum discord is plotted in Fig. 1. It is clear that quantum discord is a decreasing function of acceleration. The important point is that, for all inertial nonclassically correlated cases $(p>0)$, it is impossible to transfer a state to a properly classically correlated state.

For quantifying entanglement, the logarithmic negativity is evaluated for $\rho_{A, \mathrm{I}}$. Firstly, the eigenvalues of $\rho_{A, \mathrm{I}}^{\mathrm{pt}}$ are evaluated as follows

$$
\begin{aligned}
& \lambda_{1,2}\left(\rho_{A, \mathrm{I}}^{\mathrm{pt}}\right)=\frac{1}{4}\left\{1-p \cos ^{2} r \pm \sqrt{\sin ^{4} r+4 p^{2} \cos ^{2} r}\right\}, \\
& \lambda_{3,4}\left(\rho_{A, \mathrm{I}}^{\mathrm{pt}}\right)=\frac{1}{4}\left\{1+p \cos ^{2} r \pm \sin ^{2} r\right\} .
\end{aligned}
$$

Using Eq. (5), the logarithmic negativity, $N\left(\rho_{A, \mathrm{I}}\right)$, is evaluated and plotted as a function of acceleration, $r$, and fraction rate, $p$, in Fig. 2. It is clear that the entanglement of the state degrades with increasing the acceleration.

It should be mentioned that all the eigenvalues, Eq. (27), are positive except for the one appearing on the first line with a minus sign, i.e., $\lambda_{2}\left(\rho_{A, \mathrm{It}}^{\mathrm{pt}}\right)=\left(1-p \cos ^{2} r-\sqrt{\sin ^{4} r+4 p^{2} \cos ^{2} r}\right) / 4$. In the inertial limit, $r=0$, this eigenvalue is negative for $p>1 / 3$, and it is an increasing function of $r \in[0, \pi / 4]$. In the limit of infinite acceleration, $r=\pi / 4, \lambda_{2}\left(\rho_{A, \mathrm{I}}^{\mathrm{pt}}\right)$ is negative for $p>3 / 7$. In summary, for the region $p \in(1 / 3,3 / 7)$, whose inertial state is entangled, one may find some finite acceleration that makes the noninertial state separable. 


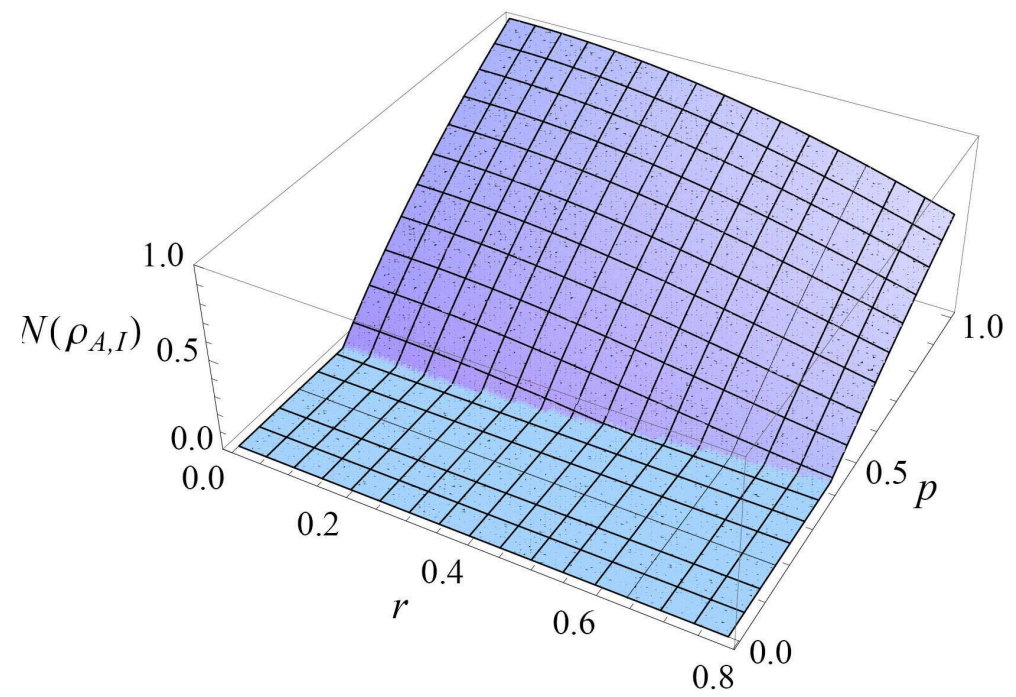

Figure 2: Logarithmic negativity, $N\left(\rho_{A, \mathrm{I}}\right)$ as funnction of $p$ and $r$.

As far as here the entanglement is studied for bipartite states the logarithmic negativity is enough to be evaluated. However, one may imply other measures of entanglement for investigation of the above states. One of the existing entanglement measure to be evaluated is entanglement of formation. The entanglement of formation is defined as [52]

$$
E_{f}(\rho)=-\frac{1+\sqrt{1-C^{2}}}{2} \log _{2} \frac{1+\sqrt{1-C^{2}}}{2}-\frac{1-\sqrt{1-C^{2}}}{2} \log _{2} \frac{1-\sqrt{1-C^{2}}}{2},
$$

where

$$
C(\rho):=\max \left\{0, \tilde{\lambda}_{1}-\tilde{\lambda}_{2}-\tilde{\lambda}_{3}-\tilde{\lambda}_{4}\right\}, \quad \tilde{\lambda}_{i} \geq \tilde{\lambda}_{i+1} \geq 0 .
$$

Here $\tilde{\lambda}_{i}$ are defined by the square roots of (positive) ordered eigenvalues of the operator $\rho\left(\sigma_{y} \otimes\right.$ $\left.\sigma_{y}\right) \rho^{*}\left(\sigma_{y} \otimes \sigma_{y}\right)$. Figure 3 represents the entanglement of formation for $\rho_{A, \mathrm{I}}$ and shows that the state is entangled for $p>3 / 7$ at infinite acceleration limit. The general behavior of this measure and the one for logarithmic negativity are the same, confirming our expectation, therefore, in the following we continue evaluating only the logarithmic negativity. 


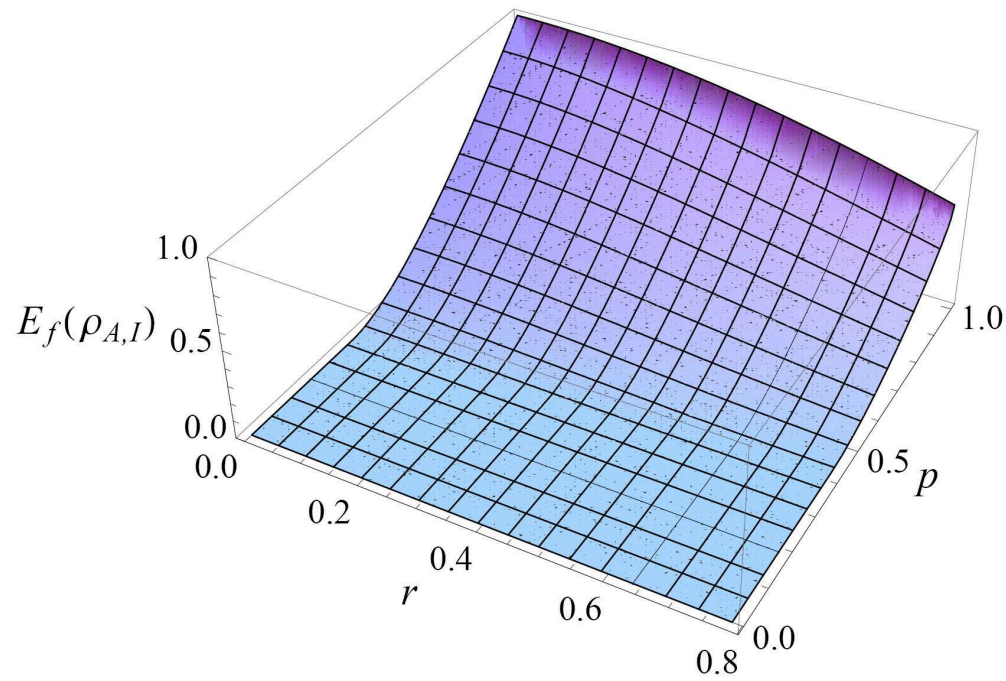

Figure 3: Entanglement of formation, $E_{f}\left(\rho_{A, \mathrm{I}}\right)$ as function of $p$ and $r$.

\subsection{Bipartition Alice-AntiRob}

Tracing out over the Rob's modes of $\rho_{A, \mathrm{I}, \mathrm{II}}$, the relating density matrix for the bipartite Alice and antiRob is calculated as

$$
\rho_{A, \mathrm{II}}=\frac{1}{4}\left(\begin{array}{cccc}
1+\cos ^{2} r-p \sin ^{2} r & 0 & 0 & 0 \\
0 & (1+p) \sin ^{2} r & 2 p \sin r & 0 \\
0 & 2 p \sin r & 1+\cos ^{2} r+p \sin ^{2} r & 0 \\
0 & 0 & 0 & (1-p) \sin ^{2} r
\end{array}\right),
$$

where, we have used the basis $|0\rangle_{A}|0\rangle_{\mathrm{II}},|0\rangle_{A}|1\rangle_{\mathrm{II}},|1\rangle_{A}|0\rangle_{\mathrm{II}}$, and $|1\rangle_{A}|1\rangle_{\mathrm{II}}$. The eigenvalues of $\rho_{A, \mathrm{II}}$ are given by

$$
\begin{aligned}
& \lambda_{1,2}\left(\rho_{A, \mathrm{II}}\right)=\frac{1}{4}\left\{1+p \sin ^{2} r \pm \sqrt{\cos ^{4} r+4 p^{2} \sin ^{2} r}\right\} \\
& \lambda_{3,4}\left(\rho_{A, \mathrm{II}}\right)=\frac{1}{4}\left\{1-p \sin ^{2} r \pm \cos ^{2} r\right\}
\end{aligned}
$$

Using these eigenvalues, the von Neumann entropy of $\rho_{A, \mathrm{II}}$ is given by $S\left(\rho_{A, \mathrm{I}}\right)=-\sum_{j=1}^{4} \lambda_{j} \log _{2} \lambda_{j}$. Similarly the entropies of the reduced density matrices $\rho_{A}$ and $\rho_{\mathrm{I}}$ are obtained as $S\left(\rho_{A}\right)=1$ and $S\left(\rho_{\mathrm{II}}\right)=-\frac{\sin ^{2} r}{2} \log _{2} \frac{\sin ^{2} r}{2}-\frac{1+\cos ^{2} r}{2} \log _{2} \frac{1+\cos ^{2} r}{2}$, respectively.

Thus, quantum discord is found to be as follows

$$
\mathcal{D}(A: \mathrm{II})=\min _{\left\{\Pi_{k}\right\}}\left[S_{\left\{\Pi_{k}\right\}}(A \mid \mathrm{II})\right]-S\left(\rho_{A, \mathrm{II}}\right)-\frac{1+\cos ^{2} r}{2} \log _{2} \frac{1+\cos ^{2} r}{2}-\frac{\sin ^{2} r}{2} \log _{2} \frac{\sin ^{2} r}{2} .
$$

It can be shown that $S_{\left\{\Pi_{k}\right\}}(A \mid \mathrm{II})$ attains its minimum in the case $\kappa=\ell=1 / 2$ with $\beta=1 / 4$ (for more details see the Appendix). Using the evaluated $\min _{\left\{\Pi_{k}\right\}} S_{\left\{\Pi_{k}\right\}}(A \mid \mathrm{II})$, quantum discord is plotted in 


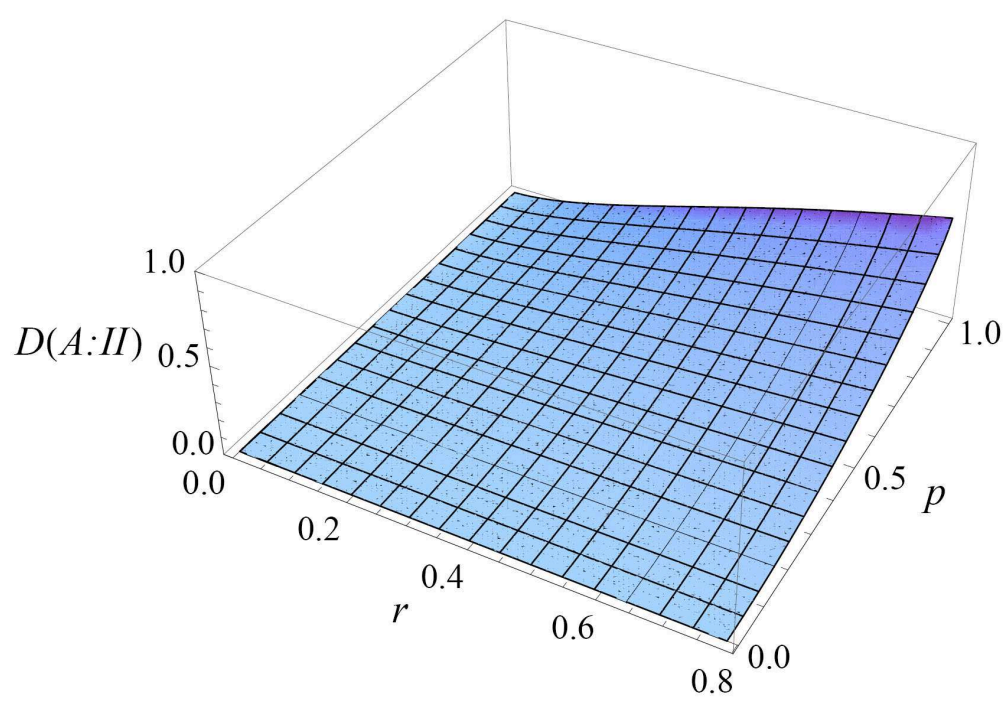

Figure 4: Quantum discord, $\mathcal{D}(A: \mathrm{II})$, as function of $r$ and $p$.

Fig. 4. It is clear that quantum discord is an increasing function of acceleration. Indeed, $\mathcal{D}(A, \mathrm{II})$ is zero for $r=0$ and it is an increasing function of acceleration (except for the case $p=0$, at which it is permanently equal to zero) and tends toward the value of $\mathcal{D}(A, \mathrm{I})$ in the limit of infinite acceleration.

The eigenvalues of $\rho_{A, \mathrm{II}}^{\mathrm{pt}}$ are given by

$$
\begin{aligned}
& \lambda_{1,2}\left(\rho_{A, \mathrm{II}}^{\mathrm{pt}}\right)=\frac{1}{4}\left\{1-p \sin ^{2} r \pm \sqrt{\cos ^{4} r+4 p^{2} \sin ^{2} r}\right\} \\
& \lambda_{3,4}\left(\rho_{A, \mathrm{II}}^{\mathrm{pt}}\right)=\frac{1}{4}\left\{1+p \sin ^{2} r \pm \cos ^{2} r\right\} .
\end{aligned}
$$

Thus, the logarithmic negativity is calculated and plotted in Fig. 5. It is clear for $r=0$, the pseudoentangled state is separable for all values of $p$. By increasing the acceleration, the state for large values of $p$ becomes entangled. for $r=\pi / 4$, the sate is entangled for $p \in[3 / 7,1]$, while it remains nonentangled for $p<3 / 7$. Similar to the previous case, one can find that the second eigenvalue, $\lambda_{2}\left(\rho_{A, \mathrm{II}}^{\mathrm{pt}}\right)=\left(1+p \sin ^{2} r-\sqrt{\cos ^{4} r+4 p^{2} \sin ^{2} r}\right) / 4$, is negative for finite values of acceleration for $p$ greater than a specific value. In the infinite acceleration limit, this eigenvalue is negative for $p>3 / 7$. Unlike the previous case, the entanglement of the state upgrades with respect to acceleration. Also, it can be verified that in the infinite limit of acceleration, the logarithmic negativity of the pseudoentangled state in Alice-Rob and Alice-antiRob bipartitions completely coincide with each other. 


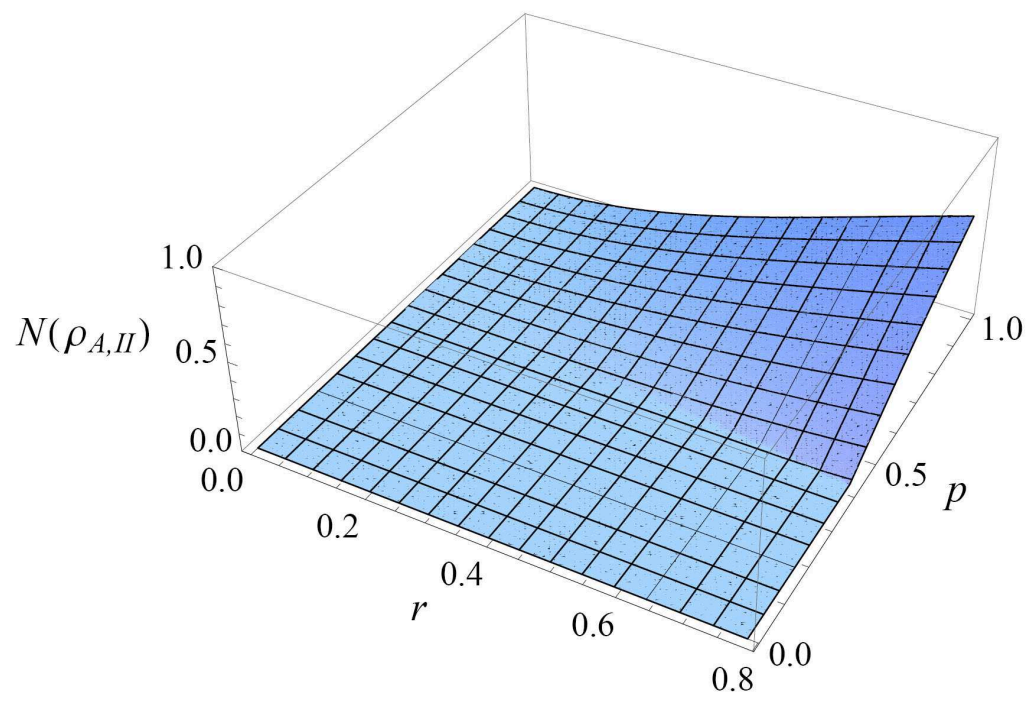

Figure 5: Logarithmic negativity, $N\left(\rho_{A, \mathrm{II}}\right)$ as function of $p$ and $r$.

\subsection{Bipartition Rob-AntiRob}

By tracing out Alice, the density matrix of Rob and antiRob is given by

$$
\rho_{\mathrm{I}, \mathrm{II}}=\frac{1}{2}\left(\begin{array}{cccc}
\cos ^{2} r & 0 & 0 & \sin r \cos r \\
0 & 0 & 0 & 0 \\
0 & 0 & 1 & 0 \\
\sin r \cos r & 0 & 0 & \sin ^{2} r
\end{array}\right) \text {, }
$$

where, we have used the basis $|0\rangle_{\mathrm{I}}|0\rangle_{\mathrm{II}},|0\rangle_{\mathrm{I}}|1\rangle_{\mathrm{II}},|1\rangle_{\mathrm{I}}|0\rangle_{\mathrm{II}}$, and $|1\rangle_{\mathrm{I}}|1\rangle_{\mathrm{II}}$. This is exactly same as the density matrix obtained for the special case $p=1$ [6, 11]. Since the entanglement of $\rho_{\mathrm{I}, \mathrm{II}}$ has been studied elsewhere [6, 11, 53], it will not be presented here. The quantum discord, $\mathcal{D}(\mathrm{I}: \mathrm{II})$, is evaluated and plotted in Fig. 7. It is shown that $\mathcal{D}$ (I : II) is an increasing function of acceleration.

\subsection{The Local Unitary Equivalent States}

One can construct the equivalent state for each states, such as the pseudo-entangled state studied in this paper, by applying local unitary operations. By operating a local operation such as $\sigma_{x}$ on the second partition of Eq. (2) we have

$$
\tilde{\rho}=\frac{1-p}{4} I+p\left|\Psi^{+}\right\rangle\left\langle\Psi^{+}\right|,
$$

where, $\left|\Psi^{+}\right\rangle=\frac{1}{\sqrt{2}}(|01\rangle+|10\rangle)$. It is an elementary exercise to check that the logarithmic negativity of $\rho$ and $\tilde{\rho}$ is the same and therefore, these two states are called the equivalent states in an inertial frame. In previous section, we considered the entanglement of $\rho$ in a noninertial frame, hence, we can do the same for $\tilde{\rho}$. Although these two density matrices are different in noninertial frame but their 


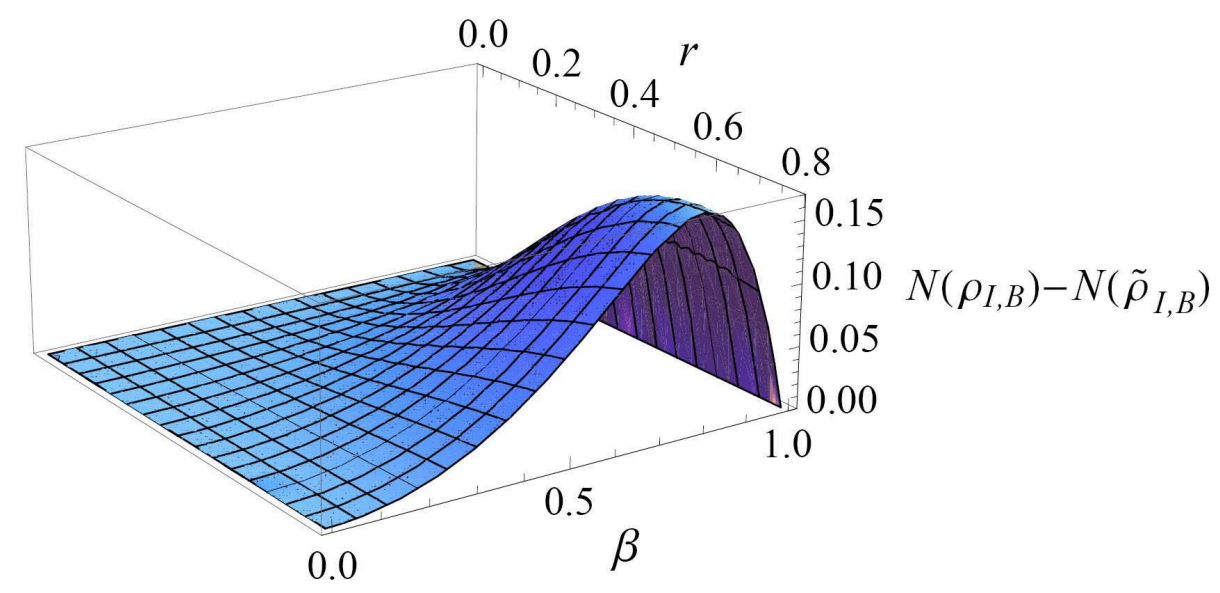

Figure 6: Logarithmic negativity, $N\left(\rho_{\mathrm{I}, B}\right)-N\left(\tilde{\rho}_{\mathrm{I}, B}\right)$ as function of $p$ and $r$.

partial transposes have the same eigenvalues and therefore, the same logarithmic negativity. In other words, these two equivalent states still remain equivalent in a noninertial frame. It is mentionable that this is not a general property.

We can investigate the behavior of $|\psi\rangle=\alpha(|00\rangle+|11\rangle)+\beta|10\rangle$ and it's equivalent $|\tilde{\psi}\rangle=\left(\sigma_{x} \otimes\right.$ $I)|\psi\rangle=\alpha(|10\rangle+|01\rangle)+\beta|00\rangle$ with $2 \alpha^{2}+\beta^{2}=1$, in a noninertial frame. If the first parties of these states are measured in an accelerating frame, similar to the previous section we can obtain $\rho_{\mathrm{I}, \mathrm{II}, B}$ $\left(\tilde{\rho}_{\mathrm{I}, \mathrm{II}, B}\right)$ and by tracing over the region II, we have the following states.

$$
\begin{gathered}
\rho_{\mathrm{I}, B}=\left(\begin{array}{cccc}
\alpha^{2} \cos ^{2} r & 0 & \alpha \beta \cos r & \alpha \cos ^{2} r \\
0 & 0 & 0 & 0 \\
\alpha \beta \cos r & 0 & \beta^{2}+\alpha^{2} \sin ^{2} r & \alpha \beta \\
\alpha \cos ^{2} r & 0 & \alpha \beta & \alpha^{2}
\end{array}\right), \\
\tilde{\rho}_{\mathrm{I}, B}=\left(\begin{array}{cccc}
\beta^{2} \cos ^{2} r & \alpha \beta \cos ^{2} r & \alpha \beta \cos r & 0 \\
\alpha \beta \cos ^{2} r & \alpha^{2} \cos ^{2} r & \alpha^{2} \cos r & 0 \\
\alpha \beta \cos r & \alpha^{2} \cos r & \alpha^{2}+\beta^{2} \sin ^{2} r & \alpha \beta \sin ^{2} r \\
0 & 0 & \alpha \beta \sin ^{2} r & \alpha^{2} \sin ^{2} r
\end{array}\right) .
\end{gathered}
$$

Figure 6 shows the differences between the logarithmic negativity of these initially equivalent states in a noninertial frame. The special cases $(\beta=0$ and $\beta=1)$ which represent the maximally entangled and separable states, respectively, still are equivalent in noninertial frame too. However, one can find a special case with $\beta \approx 0.80$ which, face with maximum differences between initial equivalent states in the infinite acceleration limit.

Finding a general description on the behavior of equivalent classes of states in noninertial frames requires further investigation and it will be explored somewhere else. 


\section{Discussion and Conclusion}

We studied the status of entanglement and nonclassical correlations for the pseudo-entangled state (2), by evaluating logarithmic negativity, $N$, and quantum discord, $\mathcal{D}$, respectively. $p$ is a representation of polarization in the physical systems such as NMR and ENDOR. In an inertial frame, if $p \leq p_{\mathrm{c}}$, where $p_{\mathrm{c}}=1 / 3$, there is no entanglement in $\rho$; however nonclassical correlations may still exist among the subsystems of $\rho$.

For the case when correlations between Alice and Rob, $\rho_{A, \mathrm{I}}$, are studied, it is shown that $N\left(\rho_{A, \mathrm{I}}\right)$ is generally decreasing as a function of acceleration and it reaches its minimum $N_{\min }\left(\rho_{A, \mathrm{I}}\right)$ for an infinite acceleration. It is also found that $p_{c}$ is increasing from $p_{\mathrm{c}}=1 / 3$ for an inertial frame up to $p_{\mathrm{c}}=3 / 7$ for an infinite acceleration, $r=\pi / 4$. This means that there is a critical range in which entanglement is destroyed due to acceleration. In other words, in noninertial frames, it can be more challenging to produce entanglement as compared to an inertial frame if $p$ falls into the critical range. Quantum discord, $\mathcal{D}(A: \mathrm{I})$, decreases from a nonzero value, for $p>0$, with respect to acceleration and it reaches its minimum $\mathcal{D}_{\min }(A: \mathrm{I})$ for $r=\pi / 4$. There is no critical range detected for discord in which its behavior is similar to that for entanglement.

Similar arguments are put forward for the partition Alice and antiRob, $\rho_{A, \mathrm{II}}$. For the stationary case, $r=0, N\left(\rho_{A, \mathrm{II}}\right)=\mathcal{D}(A: \mathrm{II})=0$, regardless of $p$. With slightly increasing acceleration, and for $3 / 7<p \leq 1, N\left(\rho_{A, \mathrm{II}}\right)$ increases up to its maximum $N_{\max }\left(\rho_{A, \mathrm{II}}\right)$ for $r=\pi / 4$. The behavior of quantum discord is simple to explain in this case as it starts increasing as a function of $r$ and for any $p>0$. It reaches its maximum value, $\mathcal{D}_{\max }(A: \mathrm{II})$, in the limit of infinite acceleration .

It is noteworthy that for $r=\pi / 4$, the logarithmic negativities of $\rho_{A, \mathrm{I}}$ and $\rho_{A, \mathrm{II}}$ approach to each other, i.e, $N_{\min }\left(\rho_{A, \mathrm{I}}\right)=N_{\max }\left(\rho_{A, \mathrm{II}}\right)$. This result is consistent with what has been reported for the special case of maximally entangled state [6]. Similarly, $\mathcal{D}_{\min }(A: \mathrm{I})=\mathcal{D}_{\max }(A: \mathrm{II})$. This has been reported, in the special case of maximally entangled state, by [11].

From the studies on the maximally entangled state, a conjecture might be that entanglement is conserved among the bipartions, Alice-Rob and Alice-antiRob. Meaning that, e.g., once the entanglement of Alice-Rob is decreasing, followingly the entanglement of Alice-antiRob is increasing. Our general study shows that entanglement cannot be conserved since at least there is a region in which the entanglement of Alice-Rob vanishes whereas the entanglement of Alice-antiRob never increases but stays in its original zero value. Note that it is clear from Eq. (33) that the entanglement of Rob and antiRob is independent of $p$.

Wang et. al. [11] picked up a maximally entangled state for their study. The state can be considered as a special case of the class of states studied in this work. Therefore, it is expected that the results of our work, for $p=1$, match with those reported in [11]. Following the method employed in [11], we have calculated $\mathcal{D}_{\mathrm{W}}(\mathrm{I}: A), \mathcal{D}_{\mathrm{W}}(\mathrm{II}: A)$, and $\mathcal{D}_{\mathrm{W}}(\mathrm{II}: \mathrm{I})$. The corresponding values are shown in Fig. 7. Employing the approach developed in this work, $\mathcal{D}(A: \mathrm{I}), \mathcal{D}(A: \mathrm{II})$, and $\mathcal{D}(\mathrm{I}: \mathrm{II})$ are calculated and shown in Fig. 7. Clearly, the corresponding values of $\mathcal{D}$ for each bipartition do 


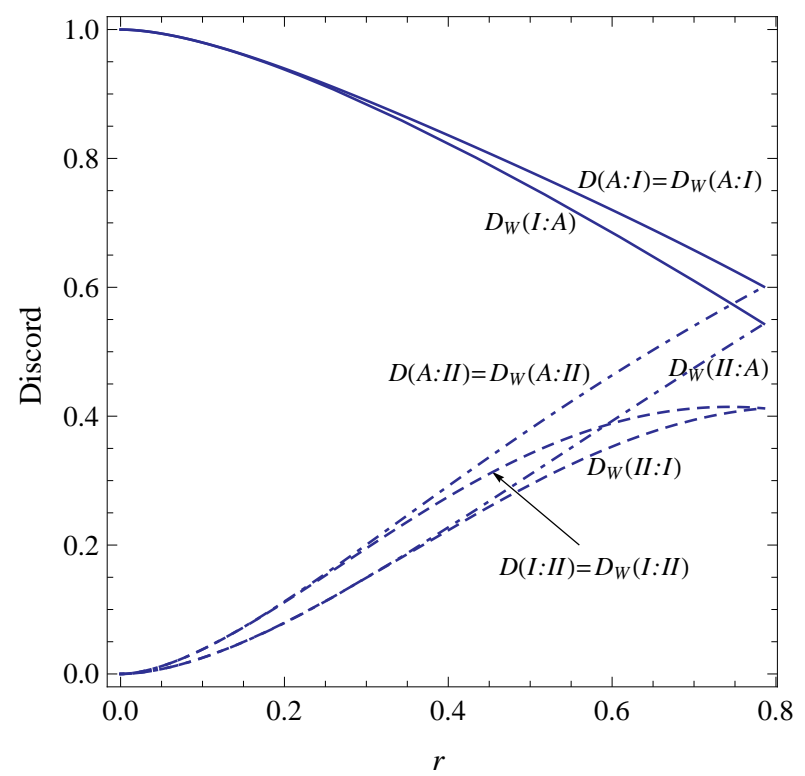

Figure 7: Quantum discords for bipartitions from Alice, Rob and antiRob as functions of $r$.

not match to each other. This inconsistency is however resolved by a simple explanation. In [11] the von Neumann projectors are applied to the first parts of bipartitions, whereas we have applied the projectors on the second parts. If the von Neumann projectors of [11] are applied to the second parts of bipartitions then $\mathcal{D}(A: \mathrm{I})=\mathcal{D}_{\mathrm{W}}(A: \mathrm{I}), \mathcal{D}(A: \mathrm{II})=\mathcal{D}_{\mathrm{W}}(A: \mathrm{II})$, and $\mathcal{D}(\mathrm{I}: \mathrm{II})=\mathcal{D}_{\mathrm{W}}(\mathrm{I}: \mathrm{II})$. Hence, a perfect consistency is observed between the results of [11], and those of ours, see Fig. 7 .

Fig. 7, is also a nice example to show the nonsymmetric behavior of quantum discord mentioned in section II as originally discussed by Zurek [47].

\section{Acknowledgment}

We would like to acknowledge one the referees for his/her valuable comments about the behavior of equivalent states in noninertial frames. We would like to acknowledge Tahmineh Godazgar for her assistance in preparing the first draft of this paper, and Ivette Fuentes for useful discussions. H. M.D. would like to acknowledge Takeji Takui for hospitality, during his visits in Osaka City University. H. M.-D. is supported by the "Open Research Center" Project for Private Universities: matching fund subsidy from MEXT. R. R. is supported by JSPS through FIRST Program, also Industry of Canada and CIFAR. 


\section{Appendix: Discord for Real Symmetric $X$-State}

Ali et. al. have evaluated [54] the quantum discord of general Hermitian $X$-shaped states represented by

$$
\rho_{A, B}=\left(\begin{array}{cccc}
\rho_{11} & 0 & 0 & \rho_{14} \\
0 & \rho_{22} & \rho_{23} & 0 \\
0 & \rho_{32} & \rho_{33} & 0 \\
\rho_{41} & 0 & 0 & \rho_{44}
\end{array}\right)
$$

For convenience, a brief review of Ref. [54] for a special real symmetric case, $\rho=\rho^{*}=\rho^{\dagger}$, is given here, which will be used for the calculations presented in this paper.

The main obstacle in evaluating quantum discord, $\mathcal{D}(A: B)$, is to obtain the minimum value of $S_{\left\{\Pi_{k}\right\}}(A \mid B)$ over all possible von Neumann measurements, i.e.,

$$
\Pi_{k}=I \otimes V|k\rangle\langle k| V^{\dagger}, \quad k=0,1 .
$$

Here, $V$ is a general $S U(2)$ element that can be written as [36, 54]

$$
V=t I+i \vec{y} \cdot \vec{\sigma}, \quad t, y_{1}, y_{2}, y_{3} \in \mathbb{R}
$$

with $t^{2}+\vec{y} \cdot \vec{y}=1$. By performing measurement $\Pi_{k}$, the state collapses to the density matrix $\rho_{A \mid k}$ with the probability $p_{k}$. One can easily find $p_{k}$ as

$$
p_{0}=1-p_{1}=\left(\rho_{22}+\rho_{44}\right) \ell+\left(\rho_{11}+\rho_{33}\right) \kappa,
$$

with $\ell=1-\kappa=y_{1}^{2}+y_{2}^{2} \in[0,1]$. The eigenvalues of $\rho_{A \mid k}$ can be obtained [54] as

$$
\lambda_{ \pm}\left(\rho_{A \mid k}\right)=\frac{1}{2}\left(1 \pm \theta_{k}\right)
$$

where, $\theta_{k}$ are given by

$$
\begin{aligned}
& \theta_{0}=\frac{1}{p_{0}} \sqrt{\left[\left(\rho_{11}-\rho_{33}\right) \kappa+\left(\rho_{22}-\rho_{44}\right) \ell\right]^{2}+\beta} \\
& \theta_{1}=\frac{1}{p_{1}} \sqrt{\left[\left(\rho_{11}-\rho_{33}\right) \ell+\left(\rho_{22}-\rho_{44}\right) \kappa\right]^{2}+\beta}
\end{aligned}
$$

where, $\beta=4 \kappa \ell\left(\rho_{14}+\rho_{23}\right)^{2}-16 \mu \rho_{14} \rho_{23}$, with $\mu=\left(t y_{1}+y_{2} y_{3}\right)^{2} \in[0,1 / 4]$. Having determined the eigenvalues of $\rho_{A \mid k}$, one can easily evaluate $S_{\left\{\Pi_{k}\right\}}(A \mid B)$ along the following line

$$
S_{\left\{\Pi_{k}\right\}}(A \mid B)=p_{0} S\left(\rho_{A \mid 0}\right)+p_{1} S\left(\rho_{A \mid 1}\right),
$$

where, $S\left(\rho_{A \mid k}\right)=-\sum_{ \pm} \lambda_{ \pm}\left(\rho_{A \mid k}\right) \log _{2} \lambda_{ \pm}\left(\rho_{A \mid k}\right)$.

It is clear that $S_{\left\{\Pi_{k}\right\}}(A \mid B)$ is an even function of $\kappa-\ell$ and, hence, it attains a minimum value at the middle point $\kappa=\ell=1 / 2$ or at the end points $\kappa=1-\ell=0,1$ (at which $\mu=0$ ) [54]. In the case of $\kappa=1 / 2, S\left(\rho_{A \mid 0}\right)=S\left(\rho_{A \mid 1}\right)$ and the minimization of $S_{\left\{\Pi_{k}\right\}}(A \mid B)$ is equivalent to the minimization of $S\left(\rho_{A \mid 0}\right)$. Since $\beta$ is a linear function of $\mu$, it can be shown that $\theta_{0}$, and thereby $S\left(\rho_{A \mid 0}\right)$ attain their minimum values at one of the end points $\mu=0,1 / 4$. Now, by checking all the above mentioned cases, $\min _{\left\{\Pi_{k}\right\}}\left[S_{\left\{\Pi_{k}\right\}}(A \mid B)\right]$ and, consequently, quantum discord can be calculated using Eqs. (7f-10). 


\section{References}

[1] A. Peres, and D. R. Terno, Rev. Mod. Phys. 76, 93 (2004).

[2] E. Schrödinger, Naturwissenschaften, 23, 807 (1935); ibid. 23, 823 (1935); ibid. 23, 844 (1935).

[3] R. Horodecki, P. Horodecki, M. Horodecki, and K. Horodecki, Rev. Mod. Phys. 81, 865 (2009).

[4] P. M. Alsing, and G. J. Milburn, Phys. Rev. Lett. 91, 180404 (2003).

[5] I. Fuentes-Schuller, and R. B. Mann, Phys, . Rev. Lett. 95, 120404 (2005).

[6] P. M. Alsing, I. Fuentes-Schuller, R. B. Mann, and T. E. Tessier, Phys. Rev. A 74, 032326 (2006).

[7] J. León, and E. Martín-Martínez, Phys. Rev. A 80, 012314 (2009).

[8] R. B. Mann, and V. M. Villalba, Phys. Rev. A 80, 022305 (2009).

[9] S. D. Bartlett, T. Rudolph, and R. W. Spekkens, Rev. Mod. phys. 79, 555 (2007).

[10] E. Martín-Martínez, and J. León, Phys. Rev. A 81, 032320 (2010).

[11] J. Wang, J. Deng, and J. Jing, Phys. Rev. A 81, 052120 (2010).

[12] M. Aspelmeyer, H. R. Böhm, T. Gyatso, T. Jennewein, R. Kaltenbaek, M. Lindenthal, G. Molina-Terriza, A. Poppe, K. Resch, M. Taraba, R. Ursin, P. Walther, and A. Zeilinger, Science 301, 621 (2003).

[13] C.-Z. Peng, T. Yang, X.-H. Bao, J. Zhang, X.-M. Jin, F.-Y. Feng, B. Yang, J. Yang, J. Yin, Q. Zhang, N. Li, B.-L. Tian, and J.-W. Pan, Phys. Rev. Lett. 94, 150501 (2005).

[14] R. Ursin, F. Tiefenbacher, T. Schmitt-Manderbach, H. Weier, T. Scheidl, M. Lindenthal, B. Blauensteiner, T. Jennewein, J. Perdigues, P. Trojek, B. Ömer, M. Fürst, M. Meyenburg, J. Rarity, Z. Sodnik, C. Barbieri, H. Weinfurter, and A. Zeilinger, Nature Phys. 3, 481 (2007).

[15] R. Ursin, T. Jennewein, J. Kofler, J. M. Perdigues, L. Cacciapuoti, C. J. de Matos, M. Aspelmeyer, A. Valencia, T. Scheidl, A. Fedrizzi, A. Acin, C. Barbieri, G. Bianco, C. Brukner, J. Capmany, S. Cova, D. Giggenbach, W. Leeb, R. H. Hadfield, R. Laflamme, N. Lutkenhaus, G. Milburn, M. Peev, T. Ralph, J. Rarity, R. Renner, E. Samain, N. Solomos, W. Tittel, J. P. Torres, M. Toyoshima, A. Ortigosa-Blanch, V. Pruneri, P. Villoresi, I. Walmsley, G Weihs, H. Weinfurter, M. Zukowski, and A. Zeilinger, Proc. Int. Astronaut. Congr. A2.1.3 (2008), arXiv:0806.0945. 
[16] A. Fedrizzi, R. Ursin, T. Herbst, M. Nespoli, R. Prevedel, T. Scheidl, F. Tiefenbacher, T. Jennewein, and A. Zeilinger, Nature Phys. 5, 389 (2009).

[17] P. C. W. Davies, J. Phys. A: Math. Gen. 8, 609 (1975); W. G. Uruh. Phys. Rev. D 14, 870 (1976).

[18] H. Ollivier, and W. H. Zurek, Phys. Rev. Lett. 88, 017901 (2001).

[19] L. Henderson, and V. Vedral, J. Phys. A: Math. Gen. 34, 6899 (2001).

[20] V. Vedral, Phys. Rev. Lett. 90, 050401 (2003).

[21] A. Datta, A. Shaji, and C. M. Caves, Phys. Rev. Lett. 100, 050502 (2008).

[22] J. A. Jones, M. Mosca, and R. H. Hansen, Nature 393, 344 (1998).

[23] R. Rahimi, K. Sato, K. Furukawa, K. Toyota, D. Shiomi, T. Nakamura, M. Kitagawa, and T. Takui, Int. J. Quantum Inf. 3, 197, (2005).

[24] D. P. Divincenzo, Forschr. Phys. 48, 771 (2000).

[25] R. F. Werner, Phys. Rev. A 40, 4277 (1989).

[26] A. Peres, Phys. Rev. Lett. 77, 1413 (1996).

[27] M. B. Plenio, and S. Virmani, Quant. Inf. Comp. 7, 1 (2007).

[28] C. H. Bennett, D. P. DiVincenzo, C. A. Fuchs, T. Mor, E. Rains, P. W. Shor, J. A. Smolin, and W. K. Wootters, Phys. Rev. A 59, 1070 (1999).

[29] J. Oppenheim, M. Horodecki, P. Horodecki, and R. Horodecki, Phys. Rev. Lett. 89, 180402 (2002).

[30] B. Groisman, S. Popescu, and A. Winter, Phys. Rev. A 72, 032317 (2005).

[31] M. Horodecki, P. Horodecki, R. Horodecki, J. Oppen-heim, A. Sen(De), U. Sen, and B. SynakRadtke, Phys. Rev. A 71, 062307 (2005).

[32] M. B. Plenio, and S. Virmani, Quant. Inf. Comp. 7, 1 (2007).

[33] M. B. Plenio, Phys. Rev. Lett. 95, 090503 (2005).

[34] B. Groisman, D. Kenigsberg, and T. Mor, arXiv:quant-ph/0703103.

[35] A. SaiToh, R. Rahimi, and M. Nakahara, Phys. Rev. A 77, 052101 (2008).

[36] S. Luo, Phys. Rev. A 77, 042303 (2008). 
[37] A. Datta, and S. Gharibian, Phys. Rev. A 79, 042325 (2009).

[38] A. SaiToh, R. Rahimi, and M. Nakahara, arXiv: 0802.2263.

[39] A. SaiToh, R. Rahimi, and M. Nakahara, arXiv: 0906.4187.

[40] R. Rahimi, and A. SaiToh, Phys. Rev. A 82, 022314 (2010).

[41] K. Modi, T. Paterek, W. Son, V. Vedral, and M. Williamson, Phys. Rev. Lett. 104, 080501 (2010).

[42] J. Cui, and H. Fan, J. Phys. A: Math. Theor. 43, 045305 (2010).

[43] M. Piani, P. Horodecki, and R. Horodecki, Phys. Rel. Lett. 100, 090502 (2008).

[44] M. Piani, M. Christandl, C. E. Mora, and P. Horodecki, Phys. Rev. Lett, 102, 250503 (2009).

[45] A. Ferraro, L. Aolita, D. Cavalcanti, F. M. Cucchietti, and A. Acin, arXiv: 0908.3157.

[46] J. Maziero, L. C. Céleri, R. M. Serra, and V. Vedral, Phys. Rev. A 80, 044102 (2009).

[47] W. H. Zurek, Phys. Rev. A 67, 012320 (2003).

[48] S. Takagi, Prog. Theor. Phys. Suppl. 881 (1986).

[49] R. Jáuregui, M. Torres, and S. Hacyan, Phys. Rev. D 43, 3979 (1991).

[50] M. Soffel, B. Müller, and W. Greiner, Phys. Rev. D 22, 1935 (1980).

[51] D. E. Bruschi, J. Louko, E. Martín-Martínez, A. Dragan, and I. Fuentes, arXiv:1007.4670.

[52] W. K. Wootters, Phys. Rev. Lett. 80, 2245 (1998).

[53] S. Moradi, Phys. Rev. A 79, 064301 (2009).

[54] M. Ali, A. R. P. Rau, and G. Alber, Phys. Rev. A 81, 042105 (2010). 